\title{
Electronic, Optical and Vibrational Properties of Bridged Dithienylethylene-Based NLO Chromophores
}

M. Carmen Ruiz Delgado, ${ }^{\S}$ Juan Casado, Victor Hernández, Juan T. López Navarrete* Department of Physical Chemistry, University of Málaga, 29071-Málaga, Spain. Jesus Orduna

Department of Organic Chemistry, ICMA, University of Zaragoza-CSIC, E-50009 Zaragoza, Spain.

Belen Villacampa, Raquel Alicante Department of Condensed Matter Physics, ICMA, University of Zaragoza-CSIC, E50009 Zaragoza, Spain.

Jean-Manuel Raimundo, Philippe Blanchard and Jean Roncali* Groupe Systèmes Conjugués Linéaires, CIMMA UMR CNRS 6200, Université d'Angers, 2 Bd Lavoisier, 49045 Angers Cedex (France).

\section{Supporting Information}

\section{Contents}

Figure S1. B3LYP/6-31G* vibrational eigenvectors associated with (a) the stretching of

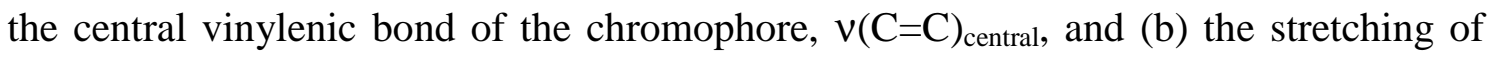
the vinylenic bond connecting the spacer and the acceptor group, $v(C=C)_{\text {acceptor }}$. Experimental and calculated (within parentheses) wavenumbers are given in $\mathrm{cm}^{-1}$.

Figure S2. Comparison between the B3LYP/6-31G* theoretical (solid line) and solid state experimental Raman spectra (dotted line) of (a) NLO-phores based on open chain DTE spacer and (b) NLO-phores based on covalently bridged DTE spacer.

\section{Complete Reference 35.}


Figure S1

(a)
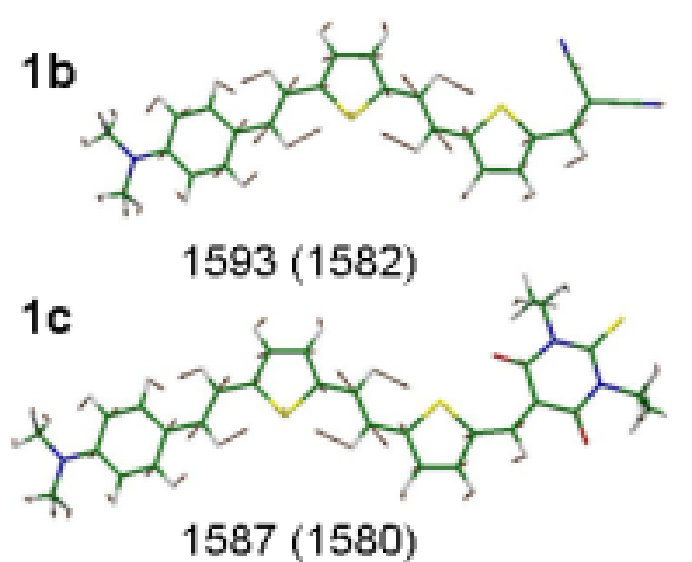

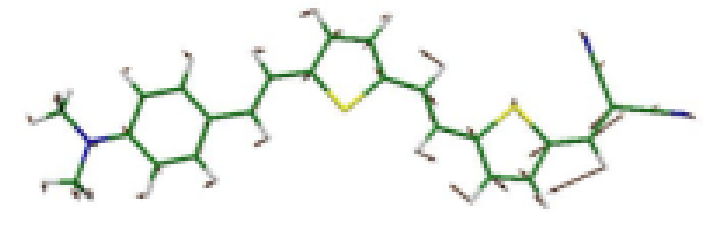

1559 (1548)

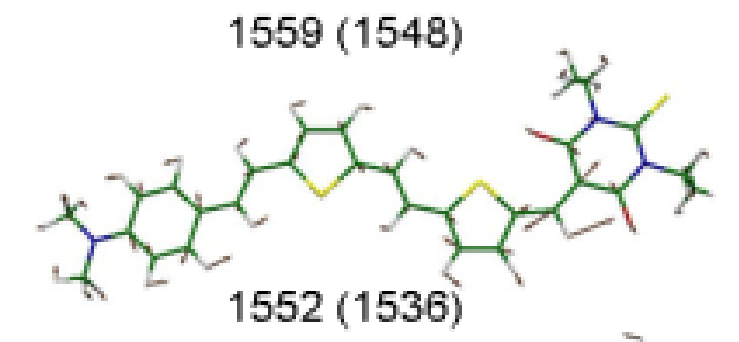

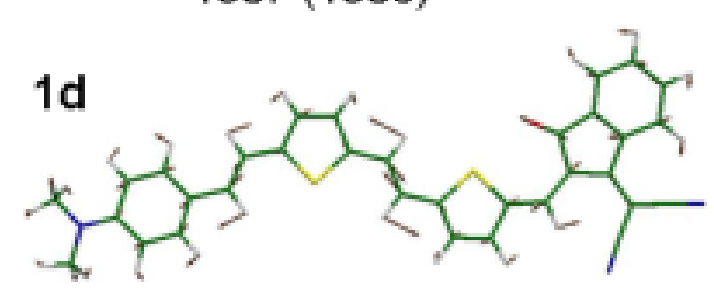

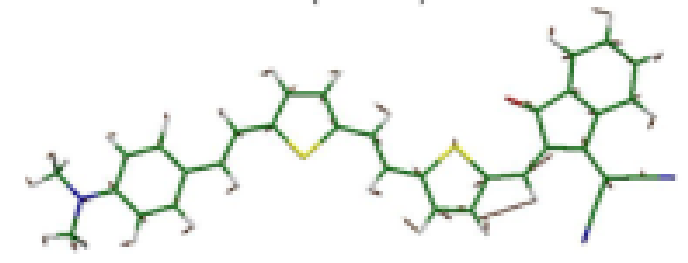

1585 (1579)
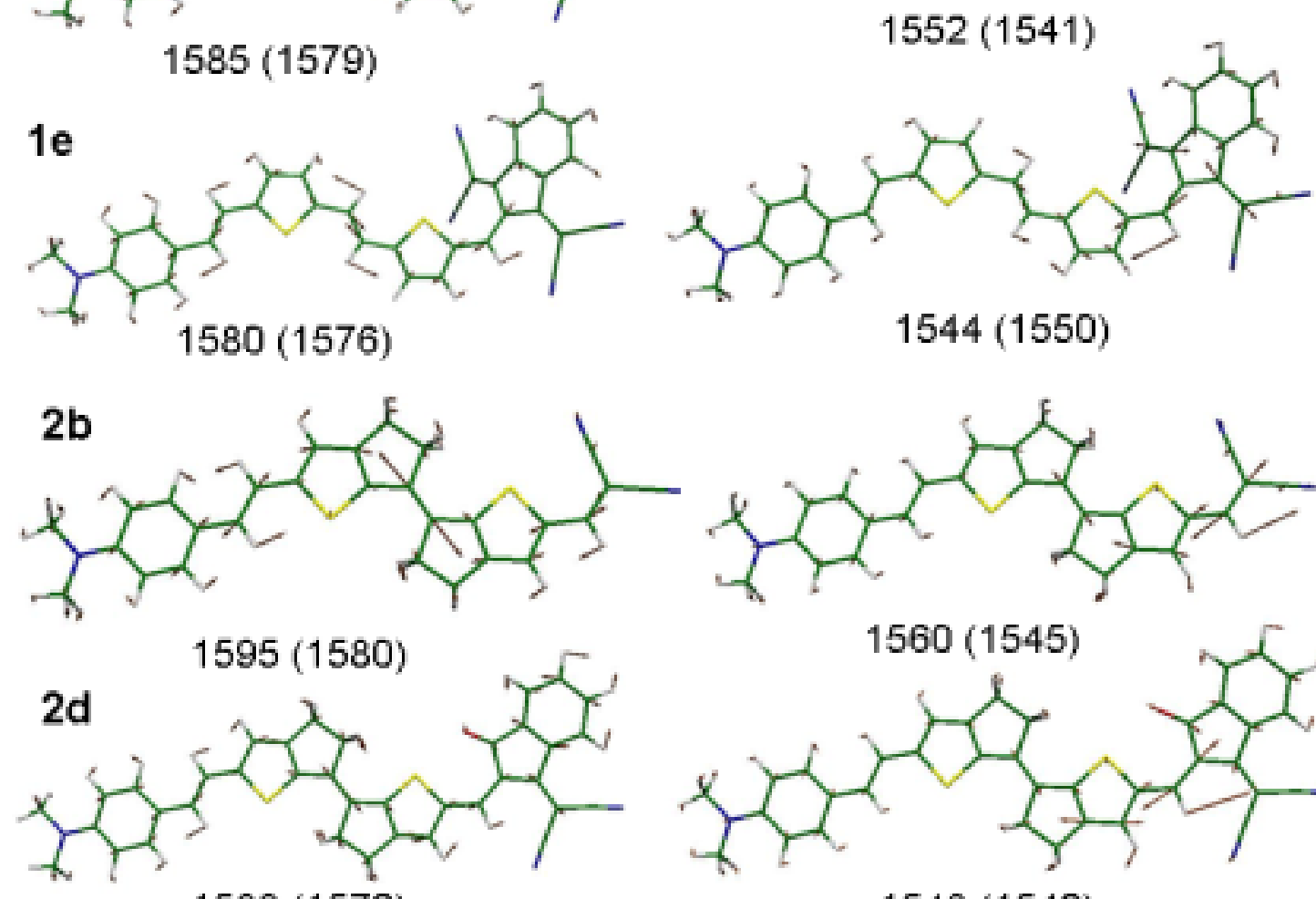

1589 (1572)
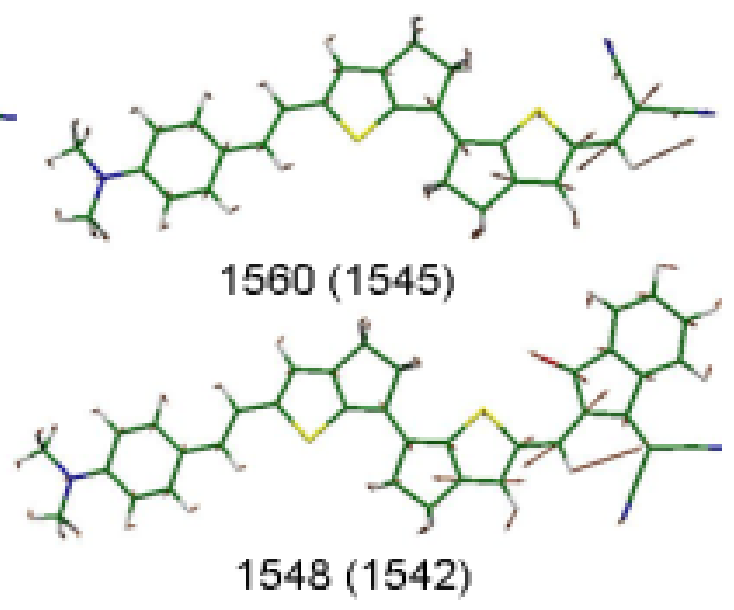
Figure S2

(a)

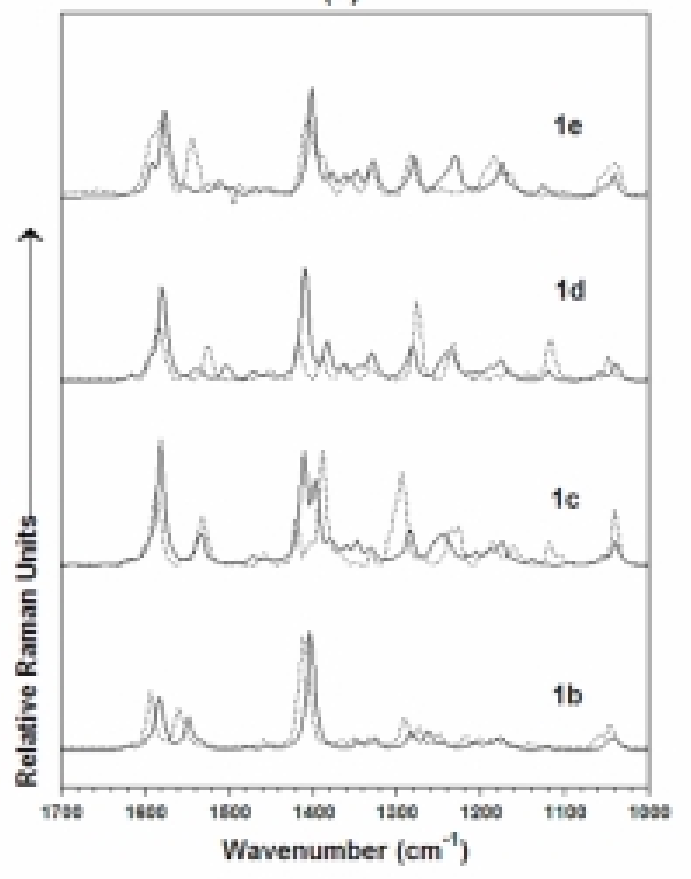

(b)

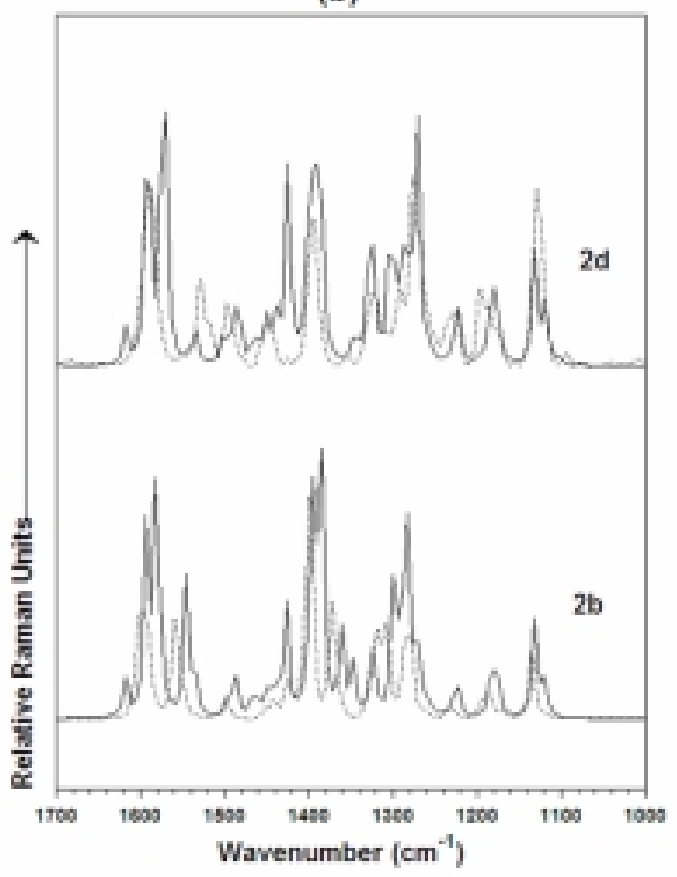




\section{Complete Reference 35.}

Frisch, M. J.; Trucks, G. W.; Schlegel, H. B.; Scuseria, G. E.; Robb, M. A.; Cheeseman, J. R.; Montgomery, J. A., Jr.; Vreven, T.; Kudin, K. N.; Burant, J. C.; Millam, J. M.; Iyengar, S. S.; Tomasi, J.; Barone, V.; Mennucci, B.; Cossi, M.; Scalmani, G.; Rega, N.; Petersson, G. A.; Nakatsuji, H.; Hada, M.; Ehara, M.; Toyota, K.; Fukuda, R.; Hasegawa, J.; Ishida, M.; Nakajima, T.; Honda, Y.; Kitao, O.; Nakai, H.; Klene, M.; Li, X.; Knox, J. E.; Hratchian, H. P.; Cross, J. B.; Bakken, V.; Adamo, C.; Jaramillo, J.; Gomperts, R.; Stratmann, R. E.; Yazyev, O.; Austin, A. J.; Cammi, R.; Pomelli, C.; Ochterski, J. W.; Ayala, P. Y.; Morokuma, K.; Voth, G. A.; Salvador, P.; Dannenberg, J. J.; Zakrzewski, V. G.; Dapprich, S.; Daniels, A. D.; Strain, M. C.; Farkas, O.; Malick, D. K.; Rabuck, A. D.; Raghavachari, K.; Foresman, J. B.; Ortiz, J. V.; Cui, Q.; Baboul, A. G.; Clifford, S.; Cioslowski, J.; Stefanov, B. B.; Liu, G.; Liashenko, A.; Piskorz, P.; Komaromi, I.; Martin, R. L.; Fox, D. J.; Keith, T.; Al-Laham, M. A.; Peng, C. Y.; Nanayakkara, A.; Challacombe, M.; Gill, P. M. W.; Johnson, B.; Chen, W.; Wong, M. W.; Gonzalez, C.; Pople, J. A. Gaussian 03, revision B.05; Gaussian, Inc.: Pittsburgh, PA, 2003. 